\title{
Inhaltsverzeichnis und Zeittafel
}

1. Vorgeschichte des Deutschen Reiches Seite

58 v. Chr. Vertreibung Ariovists aus dem Elsaß durch Cäsar. 9 n. Chr. Shlacht im Teutoburger Wald. - 90 n. Chr. ff. Anlage des Limes. - $250 \mathrm{ff}$. Die Schwaben (Alemannen) überschreiten den Limes. Auftreten der Franken am Niederrhein. - 358 Ansiedlung der salischen Franken in Nordbrabant. - 375 Zerstörung des gotischen Reiches in SüdruBland durch die Hunnen. - 382 Ansiedlung der Goten auf der Balkanhalbinsel. - $402 \mathrm{ff}$. Eroberungszüge der Westgoten. - $407 \mathrm{ff}$. Wanderung der Wandalen nach Spanien. - 410 Einnahme Roms durch die Goten. Alarich 1 . - 418 Ansiedlung der Westgoten in Südfrankreich. - 429 Gründung des Wandalenreicis in Afrika. - 436 Zerstörung des Burgunderreichs am Mittelrhein. - 449 Ansiedlung der Burgunder in Savoyen. - $455 \mathrm{ff}$. Die Franken erobern das linke Rheinufer und Moselgebiet. - 466-484 Blüte des Westgotenreichs in Südfrankreich unter K. Eurich. - 486-511 Chlodwig I., Gründer des fränkischen Reiches. - 489-493 Gründung des Ostgotenreichs in Italien durch Theoderich d. Gr. - $500 \mathrm{ff}$. Einwanderung der Baiern aus Böhmen. - 507 Chlodwig siegt bei Vouglé, erobert das Westgotische Reich bis zur Garonne. - 526 Theoderich d. Gr. T. - 531/532 Eroberung des burgundischen und des thüringischen Reichs durch die Franken. - 533 Zerstörung des Wandalenreichs durch Belisar. - 536-553 Zerstörung des Ostgotenreichs durch Belisar und Narses. - 561-613 Bürgerkriege im fränkischen Reich. - 567 Abtretung der Gascogne durch die Westgoten (in Spanien) an die Franken. - 640 Pipin I. (der Altere), frănkischer Hausmeier $¥$. - 641 Arnulf, B. von Metz, Stammvater des karolingischen Hauses $\Psi$ - 687 Pipin II. (der Mittlere) siegt bei Testri, Hausmeier im ganzen Reich. - 714-741 Karl Martell Hausmeier. - 719 Bonffatius beginnt die Mission bei Hessen und Thüringem. - 732 Karl Martell schlägt die Araber zwiscien Tours und Poitiers. - 741-752 Pipin III, (der Kleine) Hausmeier. - 742 Bonifatius beginnt die Reform der fränkischen Kirche. - 744 Einverleibung des Herzogtums Schwaben. - 752-768 Pipin König. - 754 Erster Feldzug Pipins gegen die Langobarden in Italien zum Shutze Roms. - Bonifatius $\Psi$. 756 Zweiter Feldzug in Italien. Gründung des Kirchenstaats. 760-768 Unterwerfung von Aquitanien (südlich der Loire). 768-814 Karl der GroBe. 772-804 Unterwerfung der Sachsen. 774 Eroberung des langobardischen Reichs. - 778 Unglüdklicher Feldzug nach Spanien. - 788 Absetzung Herzog Tassilos, Einverleibung Bayerns. - 791-805 Zerstörung des Reichs der Avaren. - 793 ff. Eroberung von Katalonien. - $800 \mathrm{Karl}$ wird römischer Kaiser. - 814-840 Ludwig I. der Fromme. - 827 Die Araber setzen sich in Sizilien fest. - 829-843 Bürgerkriege der Könige um die Verteilung des Reichs. - 834 Beginn der Raubzüge der Normannen (Dänen). - 841 Schlacht bei Fontenoy: Kaiser Lothar I. ( 1 855) von Ludwig dem Deutschen ( 876 ) und Karl dem Kahlen ( 1 877) geschlagen, - 843 Vertrag von Verdun: Teilung des Reichs zwischen Lothar I., Ludwig dem Deutschen, Karl dem Kahlen. - $844-875$ Kaiser Ludwig II. in Italien. - 
Seite

846 Rom von den Arabern überfallen. - 869 Tod König Lothars II. von Lotharingien. - 870 Vertrag von Meerssen: Lotharingien geteilt zwischen Ludwig und Karl. - 875-877 Karl der Kahle römischer Kaiser. - 879 Karl III. König der Langobarden, 881 römischer Kaiser, vereinigt 885 das ganze Refch. Boso von Vienne König der Provence. - 880 Vertrag von Ribémont. 887 Karl III. in Deutschland abgesetzt. - 887-899 Arnulf von Kärnten. - 888 Tod Karls III. Zerfall des Reichs. Bildung des Königreichs Burgund. $-890 \mathrm{ff}$. Festsetzung und Ausbreitung der Ungarn an der Donau. - 891 Wido von Spoleto römischer Kaiser, $\$ 894$. - 896 Arnulf römischer Kaiser. - $898 \mathrm{ff}$. Kampf um Italien zwischen Ludwig von der Provence und Berengar von Friaul. - 900-911 Ludwig IV. das Kind. - 907 Niederlage der Bayern durch die Ungarn. - 910 Sieg der Ungarn bei Augsburg.

\section{Deutsches Königtum und römisches Kaisertum}

911-1056

911-918 Konrad I. Lotharingien wird französisch. - 915 Berengar I. von Italien römischer Kaiser. - 916 Synode in HohenAltheim. - 919-936 Heinrich I. - 919/920 Erstes Vorkommen des Namens regnum teutonicum. - 923-925 Unterwerfung Lotharingiens. - 924 Waffenstillstand mit den Ungarn. - 929 Schlacht bei Lenzen. Die Wenden unterwerfen sich. - 932-954 Alberich II. Patricius der Römer. - 933 Sieg über die Ungarn in der Rieth. - 934 Dänemark unterwirft siơ. - 936-973 Otto I. 937 Aufstand Thangmars. Empörung und Unterwerfung Bayerns. Die Ungarn in Süddeutschland. - 938 Die Ungarn in Sachsen. 939/940 Aufstand Heinrichs, Eberhards von Franken, Friedrichs von Mainz und Giselberts von Lothringen in Bunde mit Ludwig von Frankreich. Siege Ottos bei Birten und Andernach und Feldzug nach Frankreich. - 940 Einnahme von Brandenburg. - 942 Die Ungarn bei Wels besiegt. - 944 Konrad der Rote Herzog von Lothringen. - 945 Berengar II, als König von Italien deutscher Vassall. - 946 Feldzug nach Frankreich. - 947-950 Böhmen endgültig unterworfen und dem Reich einverleibt. - 947 Heinrich Herzog von Baiern. - 948 Gründung der Bistümer Brandenburg und Havelberg. - 949, 950 Feldzüge nach Frankreich. - 950 Ludolf Herzog von Schwaben. Vereinigung von Burgund und Provence. - 951 Feldzug gegen Berengar II. Otto König der Langobarden. - 952 Friede zu Augsburg: Berengar als Vasall wieder eingesetzt. - 953 Aufstand Ludolfs und Konrads von Lothringen. Brun von Köln Herzog in Lothringen. 954 Wilhelm Erzbischof von Mainz. Einfall der Ungarn. - 955 Schlacht auf dem Lechfeld: Ende der ungarischen Gefahr. Oktavian, seit 954 Patricius der Römer, wird Papst Johann XII. 960 Johann XII. ruft Otto gegen Berengar II. zu Hilfe. 961-963 Erster Römerzug. - 962 Otto I. Kaiser der Römer. 966-972 Zweiter Römerzug. Strafgericht in Rom. Huldigung der langobardischen Fürsten in Unteritalien. Feldzug gegen die Griechen. - 968 Otto II. Mitkaiser. Gründung des Erzbistums Magdeburg. - 972 Anerkennung Ottos als Kaiser durch die Griechen. - 973-983 Otto II. - 974-978 Kampf um Schwaben und Bayern gegen Heinrich den Zänker. Kărnten Herzogtum. 978/980 Krieg gegen Frankreich (Uberfall in Aachen 978, Feld- 
zuq bis vor Paris 979, Friede 980). - 981 Otto II. in Rom. - 982 Feldzug gegen die Araber in Kalabrien. Niederlage bei Cotrone (le Colonne). - 983 Großer Aufstand der Wenden. Otto II. in Rom. - 983-1002 Otto III. - 983-991 Regentschaft der Kaiserin Theophanu, der Kaiserin Adelheid 991-995. - 995 Römerzug. Einsetzung Papst Gregors V. (Bruns von Kärnten). - 996 Kaiserkrönung. Ungarn wird christliçes Königreich. - 998 2. Römerzug. Aufstand und Hinrichtung des Crescentius. 999-1003 Papst Silvester II. (Gerbert von Aurillac). - 1000 Ottos III. Wallfahrten nach Gnesen (Stiftung des polnischen Erzbistums) und Aachen. - 1001 3. Römerzug. Aufstand der Römer. - 1002-1024 Heinrich II. - 1002-1015 Hartwin (Arduin) von Ivrea König in Italien. - 1004 Heinrich Ir. in Mailand gekrönt. - 1014 Heinrich II. römischer Kaiser. - 1018 Friede zu Bautzen mit Boleslaw von Polen. - 1022 Feldzug nach Unteritalien gegen die Griecten. - 1024-1039 Konrad II. - 1025 Boleslaw König von Polen stirbt. - 1026 Konrad II. unterwirft Italien. 1027 Kaiserkrönung. König Heinridh III. Herzog von Bayern. 1027-1030 Aufstand Herzog Ernsts von Schwaben. - 1031 Feldzug gegen Ungarn. Verzidht auf die Leithagrenze. - $1032 \mathrm{König}$ Rudolf III. von Burgund ₹. - 1033 Myssiko von Polen unterwirft sich und legt den Königstitel ab. - 1033/1034 Eroberung von Burgund. - 1035 Vasallenaufstand in der Lombardei. 1037 Aufstand Erzbischof Ariperts von Mailand. Gesetz über Erblichkeit der Lehen. - 1038 Einschreiten in Unteritalien. Seuche und Rüdkkehr nach Deutschland. - 1039-1056 Heinrich III. 1040 Gottesfrieden in Burgund und Südfrankreich. - 1044 Feldzug nach Ungarn. Sieg an der Raab. Wiederherstellung der Leithagrenze. Beginn der Kämpfe um Lothringen gegen Gotfried den Bärtigen. - 1045 Gregor VI. Papst. - 1046 Synode in Sutri. Absetzung Gregors VI. Erhebung Clemens II. (Suidgers von Bamberg). Kaiserkrönung. - 1047 Belehnung normännischer Ritter mit Fürstentümern in Unteritalien. - Damasus II. (Poppo von Brixen), - 1049-1054 Leo IX. (Brun von Toul). Beginn der Kirchenreform. - 1053 Leo IX. im Feldzug gegen die Normannen bei Civitate gefangen. - 1054-1057 Viktor II. (Gebhard von Eidhstătt). - 1054 Gotfried der Bärtige in Toskana.

3. Das kirchliche Zeitalter . . . . . . . . . . .

1056-1106 Heinrich IV. - 1057-1058 Stefan IX. (Friedrich von Lothringen). - 1058-1061 Nikolaus II. - 1059 Synode in Rom. Investiturverbot. Ordnung der Papstwahl. Vertrag des Papstes mit den Normannen, Belehnung Robert Guiscards mit Apulien, Kalabrien und Sizilien. - 1061-1073 Alexander II. (Anselm von Lucca). - 1061-1064 Kadaloh (Honorius II.) Gegenpapst. 1062 Entführung Heinrichs IV. in Kaiserswerth. $\rightarrow 1063$ Siegreicher Feldzug nach Ungam, König Salomo deutscher Vasall. - 1064 Synode in Mantua. - 1066 Sturz Adalberts von Bremen. - 1069 Gotfried der Bärtige + - 1071 Sturz Ottos von Nordheim. - 1072 Adalbert von Bremen $T$. Petrus Damiani $¥$. In Kloster Hirsau die Ordnungen Chunys eingeführt. Robert Guiscard beendet die Eroberung von Apulien und Kalabrien, nimmt Palermo. - 1073-1075 Aufstand der Sachsen u. Thüringer. 1075 Sieg Heinrichs IV. bei Homburg an der Unstrut. -1073 
bis 1085 Gregor VII. (Hildebrand). - 1075 Dictatus papae Gregors VII. - 1076 Januar: Synode in Worms und Piacenza: Absetzung Gregors VII. Tod Herzog Gotfrieds von Lothringen. Februar: Synode in Rom; Heinrich IV. gebannt und abgesetzt. Erneuter Aufstand in Sachsen. - Oktober: Abfall der Fürsten vom König in Oppenheim. - 1077 27. Januar Lossprechung Heinrichs IV. in Cnnossa. 15. März Rudolf von Rheinfelden zum Gegenkönig gewãhlt. $\longrightarrow 1078$ Sieg Heinrichs bei Mellrichstadt. - 1079 Friedrich von Staufen Herzog von Schwaben. - 1080 Januar: Niederlage Heinrichs bei Flarchheim. März: Zweite Absetzung Heinrichs IV. Mai-Juni: Synoden in Mainz und Brixen: Absetzung Gregors VII., Wahl Wiberts von Ravenna (Clemens III.). Schenkung Mathildens von Toskana an St. Peter. 15. Oktober: Schlachten bei Volta und Hohenmölsen. Rudolf von Rheinfelden T. - 1081-1088 Gegenkönig Hermann von Salm-Luxemburg. - 1081-1084 Heinrich IV. in Italien. - 1084 Einnahme von Rom. Kaiserkrönung Heinrichs IV. Befreiung Gregors VII. durch die Normannen. Gründung des Karthäuserordens. - 1085 Gregor VII. I in Salerno (25. Mal) Robert Guiscard *. 1088-1099 Urban II. (Odo von Chátillon). - 1089 Welf von Bayern heiratet Mathilde von Toskana. - 1090 Heinrich IV. nach Italien. - 1093 Bündnis lombardischer Städte, Gegenkönig Konrad. Heinrich IV. in Verona eingeschlossen. - 1095 Synode in Clermont: Aufruf zum Kreuzzug. - 1096 Trennung Welfs von Mathilde - 1097 Rückkehr des Kaisers nach Deutschland. 1099 Einnahme Jerusalems durd die Kreuzfahrer. - 1099-1118 Paschalis II. - 1100 Gegenpapst Wibert F. - 1101 Gegenkönig Konrad $¥$. - 1104 Aufstand Heinrichs V. - 1105 Herzog Friedrich von Schwaben 1. - 1105 Abdankung des Kaisers. - 1106 bis 1125 Heinrich V. - 1107 Konkordat von Canterbury. 1110/1111 Römerzug Heinricts V. - 1111 12. Februar: MiBlungener Vertrag mit dem Papst. 12. April Kaiserkrönung und Investiturprivileg. - 1112 Mărz: Synode in Rom erklärt das Privileg für ungültig. Aufstănde in Deutschland. - 1115 Februar: Niederlage des Kaisers am Welfesholz. Sieg Ottos von Ballenstädt über die Wenden bel Köthen. Mathilde von Toskana 4. Heinrich V. nach Italien. - 1115 Bernhard Abt von Clairvaux. - 1118/1119 Gelasius II. Gegenpapst Mauritius von Braga (Burdinus, Gregor VIII.). - 1119-1124 Calixt II. - 1119 Verhandlungen zwischen Kaiser und Papst in Mouzon. - 1120 Gründung des Templerordens, der Prämonstratenser und Johanniter. - 1122 23. September: AbschluB des Konkordates in Worms. - 1123 1. Lateransynode. - 1124 Geplanter Krieg gegen Frankreich. - 1125-1137 K. Lothar. - 1127-1134 Herzog Konrad von Franken Gegenkönig. - 1127 Roger II Graf von Sizilien gewinnt auch das Festland von Unteritalien, nimmt den Königstitel an. - 1129-1130 Einnahme von Nūrnberg und Speyer durch Lothar. - 1130 Zwiespältige Papstwahl: Anaklet II. (1130-1138) und Innozenz II. (1130-1143). - 1131 Lothar und Innozenz II. in Lüttich. - 1132/1133 Römerzug und Kaiserkronung Lothars. Belehnung mit der Erbschaft Mathildens von Toskana. - 1134 Einnahme von Ulm. Friede mit den Staufern. - Albrecht der Bär, Markgraf der Nordmark, erobert Havelberg. - 1136 Lothar in Italien. Unterwerfung der lombardischen Städte. - 1137 Feldzug gegen Sizilien, Einnahme von Bari und Salerno. Albrecht der 
Bãr erobert die Priegnitz. - 1138-1152 Konrad III. - 1138 bis $1142 \mathrm{Krieg}$ um Bayern und Sachsen (Welfen gegen Babenberger). - 1139 Heinrich del Stolze von Bayern $\Psi$. Innozenz II. bei San Germano gefangen und zur Anerkennung Rogers II. gezwungen. - 1140 Einnahme von Weinsberg. - 1142 Friede zu Frankfurt: Heinrich der Löwe behălt Sachsen, Heinrich von Osterreich Bayern. - 1143 Gründung von Lübeds durch Adolf von Holstein. Aufstand der Stadt Rom gegen den Papst. - 1144 Edessa von den Türken erobert. Albrecht der Bär Markgraf von Brandenburg. - 1145-1153 Eugen III. Rom freie Republik. Arnold von Brescia. - 1147-1149 Kreuzzug Konrads III. - 1147 Kreuzzug gegen die Wenden. - 1149/50 Aufstand der Welfen. -

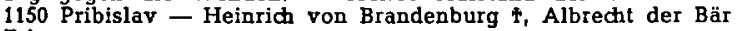
Erbe.

\section{Wiederherstellund und Untergang des Kaisertums .}

1152-1190 Friedrich I. - 1153 Besetzung des Erzbistums Magdeburg nach dem Willen des Königs. Bernhard von Clairvaux $t$. Vertrag von Konstanz zwischen Kaiser und Papst. Klage von Lodi gegen Mailand. Heinrichs des Löwen Anspruch auf Bayern anerkannt. Die Kirchen im Kolonialgebiet ihm überlassen. 1154 Friedrich in Oberitalien. Zerstörung von Tortona, Acht über Mailand. - 1154-1159 Hadrian IV. - 1154-1166 Wilhelm I. König von Sizilien. - 1155 Arnold von Brescia hingerichtet. Kaiserkrönung. - 1156 Reichstag in Regensburg: Heinrich der Löwe erhält Bayern, Osterreich wird Herzogtum. Friedrich heiratet Beatrix von Burgund. Vertrag von Benevent zwischen Hadrian IV. und Sizilien. - 1157 Feldzug gegen Polen. Reichstag in Besançon: Zusammensto B mit der römischen Kirche. - 1158 Feldzug nach Italien. Unterwerfung Mailands. Reichstag von Roncaglia; Wiederherstellung der kaiserlichen Rechte in Italien. - 1159 Empörung und Belagerung Mailands. Zwiespältige Papstwahl: Alexander III. (1159-1181) und Viktor IV. - 1160 Synode in Pavia. - 1162 Ubergabe und Zerstörung von Mailand. Gescheiterte Zusammenkunft Friedridss mit Ludwig VII. von Frankreich. 1163 Schlesien mit Deutschland vereinigt. - 1164 Bündnis von Verona u. a. mit Venedig und Konstantinopel. - 1165 Vertrag Friedrichs I. mit England. Vereidigung im ganzen Reich gegen Alexander III. - 1166-1189 Wilhelm II. König von Sizilien. - 1167 Friedrich 1 . in Italien. Feldzug gegen Rom und Sizilien. Ankona den Griechen wieder entrissen. Bündnis von Cremona u. a., Wiederaufbau von Mailand. Deutscher Sieg über die Römer bei Tuskulum. Rom nimmt den Kaiser auf, erhält Selbstverwaltung als kaiserlicie Stadt. Seuche und Rüdkzug. Reinald von Köln $\Psi$. 1. Dezember: Gründung der lombardischen Liga. - 1168 Rüdkkehr des Kaisers nach Deutschland. Lebensgefahr in Susa. - 1174 Friedrich wieder in Italien. Belagerung von Alessandria. - 1175 Friede von Montebello. Erneuter Krieg. - 1176 Friedris I. und Heinrich der Löwe in Chiavenna. 29. Mai: Schlacht bei Legnano. Okt.: Vorfriede mit Alexander III. in Anagni - 1177 Friede von Venedig. - 1178 Rüdkkehr des Kaisers nach Deutschland. - 11793 3. Laterankonzil. Heinrich der Löwe geächtet. - 1180 Absetzung Heinrichs des Löwen. Teilung des Herzogtums Sachsen. Bayern an Otto von Wittelsbach 
Steiermark Herzogtum. - 1181 Unterwerfung Heinrichs des Löwen. - 1183 Friede von Konstanz. - 1184 Schwertleite der Kaisersöhne in Mainz. - Zusammenkunft des Kaisers mit Lucius III. in Verona. Friede mit Sizilien. - 1185-87 Urban III. Bruch mit dem Kaiser. - 1186 Hochzeit Heinrichs VI. mit Konstanze von Sizilien in Mailand. Unterwerfung des aufständischen Cremona. Gründung des Klosters Oliva bei Danzig. - 1187 Saladin erobert Jerusalem. - 1188 Reichstag Jesu Christi in Mainz. - 1189 Kreuzzug Friedrichs I. - Aufstand Heinrichs des Löwen. Wilhelm II. von Sizilien 1. - 1190-97 Heinrich VI. - 1190 Waffenstillstand mit Heinrich dem Löwen. - 1191 Erster Feldzug gegen Sizilien. Kaiserkrönung. Belagerung von Neapel und Rückkehr. - 1192 Erhebung der Welfen und niederländischer Fürsten. Gefangennahme Richards I. von England. - 1194 Befreiung Richards. Friede im Reich, Unterwerfung der Welfen. Eroberung Siziljens. 11. Dez.: Friedridh II, geboren. - 1195 Heinrich der Löwe $\Psi$. Rückkehr des Kaisers aus Italien. -- 1195/6 Erbreichsplan. - 1197 Verschwörung in Sizilien. Kreuzzug. Huldigung von Zypern und Armenien. - 28. Sept.: Heinrich VI. F. Aufstand im Königreich und ganz Italien. - 1198-1216 Innozenz III. Vergrößerung des Kirchenstaats. - 1198 Doppelwahl in Deutschland: Philipp von Schwaben und Otto IV. Der Deutsche Ritterorden gegründet. - 1199 Richard von England 1 . Albert von Bremen Bischof in Livland. - 1201 Geheimvertrag Ottos IV. mit dem Papst. Riga und der Schwertbrüderorden in Livland gegründet. - 1202-41 Waldemar II. König von Dänemark. $1203 \mathrm{ff}$. Waldemars Eroberungen im deutschen Kolonialgebiet. - 1204 Johann ohne Land unterliegt gegen Frankreich. Beginnender Abfall von Otto IV. - 1206 Philipp unterwirft Köln. - 1207 Belehnung Bischof Alberts mit Livland. -120821 . Juni: Philipp von Sdhwaben ermordet. Otto IV. allgemein anerkannt. - 1209 Otto IV. erneuert dem Papst sein geheimes Versprechen; bricht es. 4. Okt.: Ottos Kaiserkrönung. - 1210 Nov.: Otto unternimmt die Eroberung des sizilischen Reiches, wird vom Papst gebannt. - 1210-39 Hermann von Salza Meister des Deutscien Ordens. - 1211 Sept.: Friedrich von Sizilien Gegenkönig, Otto kehrt nach Deutschland zurück. - 1211-25 Der Deutsche Orden in Ungarn. - 1212 Sept. Friedrich von Sizilien in Deutschland. Abfall von Otto. - 1212-50 Friedrich II. 1213 Goldene Bulle von Eger für die römische Kirche. - 1214 27. Juli: Schladht bei Bouvines. - 1215 Friedridh II. in Aachen gekrönt, gelobt den Kreuzzug. - 1216-27 Honorius III. - 1216 Heinrich von Sizilien wird Herzog von Schwaben. - 1218 Otto IV. $\Psi$. Die Zähringer sterben aus. Gründung von Rostock. Estland wird dänisch. - 1219 Heinrich von Schwaben wird Rektor von Burgund. - 1220-35 Heinrich (VII.) deutscher König. Großes Privileg für die geistlichen Fürsten. Kaiserkrönung Friedrichs II. Gesetze zugunsten der Kirche. - 1225 Reichsregent Engelbert von Köln ermordet. Livland zur Reichsmark erklärt. 1226 Reichstag in Cremona durch die erneuerte Liga der Lombarden verhindert. Wilhelm von Modena ordnet Livland. Preußen dem Deutschen Ritterorden verliehen. - 1227-41 Gregor IX. 1227 Friedrich II. wegen Unterlassens des Kreuzzugs gebannt. 1228/9 Kreuzzug des Kaisers. 10jähriger Friede mit Agypten. Friedrich II. König von Jerusalem. Krieg zwischen Kaiser und 
Papst. - 1228 Estland wieder deutsch. - 1230 Friede von Ceprano zwischen Kaiser und Papst. Reval zuerst erwähnt. 1231 Reichstag in Ravenna durch die Lombarden verhindert. 1231/2. Großes Privileg über die Landeshoheit der Fürsten. 1232 Kulm und Thorn gegründet. - 1234/5 Aufstand und Unterwerfung Heinrichs (VII.). - 1235 Reichstag in Mainz. Landfriedensgesetz. Braunschweig zum Herzogtum erhoben. Friedrich II, heiratet Isabella von England. Reichskrieg gegen Mailand erklärt. - 1236 Untergang des Schwertritterordens an der Saule. $-1237-54$ Konrad IV. -1237 Sieg des Kaisers bei Cortenuova. Der Deutsche Orden übernimmt die Verteidigung Livlands. Elbing gegründet. - 1238 Bündnis von Genua und Venedig gegen den Kaiser. Gregor IX. greift ein. Estland an Dänemark abgetreten. - 1239 20. März: Gregor IX. bannt den Kaiser. 1241 Seesieg des Kaisers bei Monte Cristo. 1242 Die Erzbischöfe von Köln und Mainz verkünden den Bann gegen den Kaiser. - 1243-54 Innozenz IV. - 1244 Friede zwischen Papst und Kaiser. Innozenz IV. flühtet nach Lyon. - 1245 Konzil in Lyon. Absetzung des Kaisers (17. Juli). - 1246/7 Heinrich Raspe Gegenkönig. - Sieg des Gegenkönigs bei Frankfurt. Vergebliche Belagerung von Ulm. - $1247-56$ Wilhelm von Holland Gegenköníg. - 1250 13. Dez. Friedrich II. T. - $1266 \mathrm{Karl}$ von Anjou siegt bei Benevent und erobert das sizilische Reich. 1268 Konradin bei Tagliacozzo geschlagen, gefangen und hingerichtet.

Register . . . . . . . . . . . . . . . . . 149

\section{Kartenverzeichnis}

1. Der Reichskern aus der karolingischen Erbschaft um 900 . . . . . . . . . . . . . . . . . 27

2. Das Reich in seiner Höchstausdehnung um 1250 . . 29

3. Italien vor der normannischen Eroberung . . . . 103

4. Staufen und Welfen . . . . . . . . . . . . 103 